\title{
On the Robustness of Least-Squares Monte Carlo (LSM) for Pricing American Derivatives
}

\author{
Manuel Moreno \\ Department of Economics and Business \\ Universitat Pompeu Fabra \\ Carrer Ramón Trias Fargas, 25-27 \\ 08005 Barcelona, Spain \\ phone: (34-93) 5.42.27.71 \\ fax: $\quad$ (34-93) 5.42.17.46 \\ e-mail: manuel.moreno@econ.upf.es.
}

\author{
Javier F. Navas \\ Department of Finance \\ Instituto de Empresa \\ María de Molina, 12, planta 4 \\ 28006 Madrid, Spain \\ phone: (34-91) 5.68.96.00 \\ fax: $\quad(34-91) 5.63 .68 .59$ \\ e-mail: jfnavas@ie.edu
}

This Version: April 2001

Keywords: Least-Squares Monte Carlo, Option Pricing, American Options.

Journal of Economic Literature classification: C15, C60, G13.

\section{Acknowledgments}

We would like to thank E.S. Schwartz for their valuable comments and suggestions. We also thank seminar participants at the Universidad Complutense de Madrid. The first author gratefully acknowledges financial support by DGES grant PB98-1057 and the hospitality of the Financial Options Research Centre, at Warwick Business School, where part of this work was done. The usual caveat applies. 


\begin{abstract}
This paper analyses the robustness of Least-Squares Monte Carlo, a technique recently proposed by Longstaff and Schwartz (2001) for pricing American options. This method is based on least-squares regressions in which the explanatory variables are certain polynomial functions. We analyze the impact of different basis functions on option prices. Numerical results for American put options provide evidence that a) this approach is very robust to the choice of different alternative polynomials and b) few basis functions are required. However, these conclusions are not reached when analyzing more complex derivatives.
\end{abstract}




\section{Introduction}

How much do you pay for a certain asset if you know its final pay-off but you ignore when you will receive it? That is one of the main questions that academics and practitioners interested in American derivatives try to answer. The difficulty for answering this question arises because we do not know the exact time at which we will receive the reward promised by the asset and, then, there exists a possibility of early exercise. At each exercise time before maturity, the holder of this asset must decide if he exercises the option or if he waits until a future exercise date. This decision depends on the comparison, at each date, between the (known) immediate exercise value and the (unknown) continuation value.

Closed-form expressions for derivative prices exist in a few special cases. One example is an European option written on a single underlying asset whose price was derived by Black and Scholes (1973) and Merton (1973). Analytical expressions for the price of American options have been found, but there are no easily computable formulae currently available. Then, numerical methods such as trees, finite difference schemes, quadrature routines or Monte Carlo simulation are usually required.

The Monte Carlo approach simulates trajectories for asset prices. An estimation of the option price is obtained by the (discounted) average of the option cash-flows computed for each path. Monte Carlo simulation is appropriate to price options with complex features (path-dependency, multiple stochastic processes, random volatility, jumps, ...).

Although this technique is well suited for pricing European options, it has not been widely applied to American derivatives, which are priced using other numerical methods. Recently, Longstaff and Schwartz (2001) have developed an algorithm in which the continuation value is estimated appropriately by a leastsquares regression jointly with the cross-sectional information provided by Monte Carlo simulation. They name this technique Least-Squares Monte Carlo (LSM). 
Concretely, these authors propose to regress the (discounted) cash-flows expected to be received in the future against a set of (basis) functions whose arguments are the underlying asset prices. These authors claim that the choice of different functions as well as the number of terms used have little effect on the solution of the problem.

In this paper we analyze the robustness of the LSM approach for pricing American derivatives. For a put option, we find that LSM is indeed very robust to the choice of the basis functions and only three or four terms are usually required to obtain a reliable price. We also study more sophisticated derivatives. For the case of an option on the maximum of five assets, we find that the number of terms and the choice of functions can have non-negligible effects on option prices.

This article is organized as follows. Section 2 reviews option pricing models, focusing on American-style derivatives. In Section 3, we briefly present the LSM technique and we provide a numerical example. Section 4 describes the set of basis functions used in this paper and we study the pricing of some American derivatives. Finally, Section 5 concludes the paper.

\section{A Review of Derivative Pricing Models}

\subsection{The Standard Black-Scholes / Merton Model}

In the standard Black-Scholes / Merton model, the economy consists of an European option, its underlying asset (the stock) and a risk-free asset. The model assumes this market is frictionless, there is a continuous trading of assets and there exists a constant risk-free rate, $r$, for lending and borrowing. ${ }^{1}$ Moreover, the price of the underlying asset, $S$, is assumed to follow the GBM (risk-neutral)

\footnotetext{
${ }^{1}$ Thus, the amount $B_{t}$ invested in the risk-free asset at time $t$ follows the differential equation $d B_{t}=r B_{t} d t$
} 
process

$$
d S=r S d t+\sigma S d z
$$

where $\sigma$ is the (constant) volatility rate of the stock return and $z$ is a standard Brownian motion.

Sometimes, it is useful to reformulate this equation in terms of the natural logarithm of the asset price, $x=\ln (S)$. With this new variable, equation (1) becomes

$$
d x=\nu d t+\sigma d z, \quad \nu=r-\frac{1}{2} \sigma^{2}
$$

with the advantage that we have now a constant term in both the drift and the volatility of the stochastic process.

After building a riskless portfolio and applying no-arbitrage conditions, these authors derive the following partial differential equation (PDE) for the option price $C(S, t)$

$$
\frac{1}{2} \sigma^{2} S^{2} \frac{\partial^{2} C(S, t)}{\partial S^{2}}+r S \frac{\partial C(S, t)}{\partial S}+\frac{\partial C(S, t)}{\partial t}=r C(S, t)
$$

The initial boundary condition is given by the final pay-off of the option. For a call option, it is given by

$$
C(S, T)=\max \left\{S_{T}-K, 0\right\}
$$

If we use the variables $x=\ln (S), W(x, t)=C(S, t)$, we obtain a PDE with constant coefficients for the partial derivatives

$$
\frac{1}{2} \sigma^{2} \frac{\partial^{2} W(x, t)}{\partial x^{2}}+\nu \frac{\partial W(x, t)}{\partial x}+\frac{\partial W(x, t)}{\partial t}=r W(x, t)
$$

This PDE, after a certain change of variables, ${ }^{2}$ is equivalent to the well-known "heat equation" and its solution (the call price) is given by

$$
C(S, t)=S N\left(d_{1}\right)-K e^{-r(T-t)} N\left(d_{2}\right)
$$

\footnotetext{
${ }^{2}$ See Black and Scholes (1973) for details.
} 
where $N($.$) is the cumulative normal distribution function and$

$$
d_{1}=\frac{\ln \left(\frac{S}{K}\right)+\nu(T-t)}{\sigma \sqrt{T-t}}, \quad d_{2}=d_{1}-\sigma \sqrt{T-t}
$$

For an European put option, we can use the put-call parity to obtain

$$
P(S, t)=K e^{-r(T-t)} N\left(-d_{2}\right)-S N\left(-d_{1}\right)
$$

For an American option, the only difference is that, at each exercise time, we must decide whether to exercise the option or to wait. The boundary which separates the early exercise and the continuation regions is the optimal exercise boundary which must be determined to price the option.

\subsection{Numerical Methods for Pricing American Derivatives}

We now summarize the main techniques that have been proposed in the literature. Most of these methods are suitable just for some derivatives and there is no consensus on which of these approaches is the "winner" one. We review analytical solutions and numerical and analytical approximations.

Analytical solutions provide closed-form expressions for option prices. Although this approach is the most elegant (and fastest) method for pricing derivatives, it may happen that very strict assumptions can lead to an (empirically) unrealistic model.

Analytical solutions for the case of an American call option with discrete dividends have been derived by Roll (1977), Geske (1979) and Whaley (1981). The solution for the infinite horizon case is provided by McKean (1965). Recently, Ait-Sahlia (1996) and Ait-Sahlia and Lai (1996, 2000) have obtained closed-form expressions for the optimal exercise boundary.

Other analytical solutions have been obtained by the method of lines (see Rektorys (1982)) that is applied by Carr and Faguet (1996) and Carr (1998). Carr and Faguet (1996) discretize the time derivative in the Black-Scholes PDE and 
then solve analytically the resulting sequence of ordinary differential equations. In a similar way, Carr (1998) also discretizes the time derivative and proposes to randomize the expiration date of the American option in order to price it. He shows that this problem is equivalent to the infinite horizon case. Then, he uses the results of McKean (1965) to obtain exact prices.

Analytical approximations are closed-form solutions for approximations to the original problem. For American options, this technique has been used by Johnson (1983), Geske and Johnson (1984), Barone-Adesi and Whaley (1987), Bunch and Johnson (1992), Broadie and Detemple (1996) and Ho et al. (1997), among others. See Ait-Sahlia and Carr (1997) and Ju (1998) for a comparison of these techniques.

Johnson (1983) presents an interpolation method based on regressing option prices against lower and upper bounds for these prices. A similar technique can be found in Broadie and Detemple (1996), where the lower bound (LBA) and the average of lower and upper bound (LUBA) methods are described. The results depend strongly on the interpolation scheme and the accuracy of the bounds.

Geske and Johnson (1984) apply the Richardson extrapolation technique ${ }^{3}$ to their compound option model. They obtain an expression involving an infinite series with multidimensional cumulative normal distributions. Several modifications of this model have been suggested. Concretely, Bunch and Johnson (1992) simplify its numerical computation, Ho et al. (1994) use an exponential extrapolation and Ho et al. (1997) generalize the original technique to deal with stochastic interest rates.

Barone-Adesi and Whaley (1987) have developed a very fast approximation based on a simplification of the related PDE, but this technique is not very accurate for long maturity options.

When the assets have complex features (multiple stochastic processes, non-

\footnotetext{
${ }^{3}$ This technique has also been used to accelerate valuation methods by Breen (1991), Huang et al. (1996), Carr and Faguet (1996), Carr (1998) and Ju (1998).
} 
Markov property, ...), they cannot be priced, in general, by analytical methods. In this case, numerical methods are used.

\subsubsection{Trees / Lattices}

Lattice methods are based on the discretization of the risk-neutral processes followed by the relevant variables. Then, backward induction in time is used to solve for the option price. The most popular methods of this class are binomial and trinomial trees.

The binomial method was introduced by Cox et al. (1979) and Rendleman and Bartter (1979). The method is based on the random walk approximation to the Brownian motion and provides a simple and intuitive numerical solution.

In this method, the partition $\left\{t_{0}=0, t_{1}, t_{2}, \ldots, t_{N-1}, t_{N}=T\right\}$ of the time interval $[0, T]$ is considered. At each point of this partition, it is assumed that the price of the underlying asset follows a multiplicative binomial process: it either jumps up by a proportion $u$ or goes down by a proportion $d$. Both proportions $u$ and $d$ determine the mean and the volatility of the underlying asset.

According to this evolution of the asset price, the call option price goes either to $C_{u}=\max \{u S-K, 0\}$ or to $C_{d}=\max \{d S-K, 0\}$. As in the Black-Scholes model, a riskless portfolio is built and the price of a call option with one period to maturity is given by

$$
C=e^{-r \Delta t}\left(p C_{u}+(1-p) C_{d}\right), \quad p=\frac{e^{r \Delta t}-d}{u-d}, \quad \Delta t=T / N
$$

Thus, the call option price can be interpreted as the (discounted) expectation of the future pay-offs under the risk-neutral probabilities.

Since the binomial model is an approximation to the continuous-time model of the asset price, we choose the values of the jump parameters $(u$ and $d)$ and the (risk-neutral) probability $p$ to match the risk-neutral mean and variance of the process given in equation (1). As we have two equations and three parameters, we can freely choose one of them. Two specifications have been proposed in the 
literature: Cox et al. (1979) assume equality between jump sizes ${ }^{4}$ and Jarrow and Rudd (1983) assume equal probability of up and down jumps.

To avoid numerical problems, the change of variable $x=\ln (S)$ is recommended. Now, $x$ can go either up to $x+\Delta x_{u}$ or down to $x+\Delta x_{d}$ with probabilities $p$ and $1-p$, respectively. Equating the mean and the variance of the discrete and continuous processes, we obtain two equations and, as before, one of the three parameters $\left(\Delta x_{u}, \Delta x_{d}\right.$ or $\left.p\right)$ can be chosen in a free way.

For the specification based on $S(x)$, we can build an asset price tree starting from the initial value $S_{0}\left(x_{0}\right)$. At each node $(i, j)$ in this tree, the asset price is $S_{i, j}=S_{0} u^{j} d^{i-j}\left(x_{i, j}=x_{0}+j \Delta x_{u}+(i-j) \Delta x_{d}\right)$ and the call price is $C_{i, j}$.

We start at the final node of the tree at time $T$ where we know the value of the option (its final pay-off). Since we are in a risk-neutral framework, the value of the option at each node at time $T-\Delta t$ can be computed as the expected value at time $T$ multiplied by a discount factor

$$
C_{i, j}=e^{-r \Delta t}\left(p C_{i+1, j+1}+(1-p) C_{i+1, j}\right)
$$

and, going back through all the nodes in the tree, we obtain the value of the option at time zero, $C_{0,0}$.

For an American option, the only difference is that, at each node, we have to compare the gain obtained from early exercise with the one obtained if the option is exercised later.

Generalizations of the binomial approach have been suggested by Breen (1991) who proposes the "accelerated binomial method" with Richardson extrapolation to reduce the number of steps, and Broadie and Detemple (1996) who propose the following modifications:

1. BBS method: In the binomial model, the Black-Scholes formula replaces the "continuation value" one time step before expiration of the option.

\footnotetext{
${ }^{4}$ Under this assumption, the binomial tree is recombining, a very desirable property from a computational point of view.
} 
2. BBSR method: It consists of the BBS method plus the Richardson extrapolation technique.

The binomial model is generalized by the trinomial tree model, originally proposed by Parkinson (1977) and Boyle (1988).

This model assumes that the logarithm of the asset price, $x$, over a small interval $\Delta t$, can a) go up by $\Delta x$, b) stay the same or c) go down by $\Delta x$, with probabilities $p_{u}, p_{m}$ and $p_{d}$, respectively. As in the binomial model, the values of these probabilities are chosen to match the risk-neutral mean and variance of the process (2).

Now, an asset price tree starting from the initial value $x_{0}$ is built. Analogously to the binomial method, the value of the option at each node $(i, j)$ in this tree at time $T-\Delta t, C_{i, j}$, is computed as the discounted expected value

$$
C_{i, j}=e^{-r \Delta t}\left(p_{u} C_{i+1, j+1}+p_{m} C_{i+1, j}+p_{d} C_{i+1, j-1}\right)
$$

and backward induction leads to the current value of the option, $C_{0,0}$.

The main advantage of trinomial trees is that, for a given number of time steps, $N$, its convergence is faster than with binomial trees (although they require more memory).

\subsubsection{Finite Difference Schemes}

An alternative technique is the "finite difference" method. The first step is to introduce a grid of mesh points $(t, x)=(i k, j h), i \in Z^{+}, j \in Z$ where $h$ and $k$ are mesh parameters as small as desired. Next, an approximate solution of the PDE at these points is obtained by solving a problem in which the partial derivatives are replaced with finite differences.

Depending on whether the difference expressions are centered around time step $i+1, i$, or $i+\frac{1}{2}$, we obtain the fully explicit ${ }^{5}$, fully implicit or Crank-Nicolson

\footnotetext{
${ }^{5}$ It can be seen that this explicit method is equivalent to approximate the diffusion process by a discrete trinomial process. See Clewlow and Strickland (1998) for details.
} 
method, respectively. ${ }^{6}$

These algorithms can be compared in terms of consistency, convergence and stability properties. Intuitively, these properties can be interpreted as follows:

1. Consistency: A model is consistent when it can be as close to the original model as desired.

2. Convergence: The solution of the approximation converges to the solution of the original problem.

3. Stability: Small changes in the original conditions do not imply big changes in the results.

The following table summarizes these properties for the three methods:

\begin{tabular}{lccc} 
Method & Consistency & Convergence & Stability \\
\hline Fully explicit & $O\left((\Delta x)^{2}+\Delta t\right)$ & Only if $\Delta x>\sqrt{2 \Delta t}$ & Only if $\Delta x>\sqrt{2 \Delta t}$ \\
Fully implicit & $O\left((\Delta x)^{2}+\Delta t\right)$ & Unconditionally & Unconditionally \\
Crank-Nicolson & $O\left((\Delta x)^{2}+\left(\frac{\Delta t}{2}\right)^{2}\right)$ & Unconditionally & Unconditionally
\end{tabular}

The fully explicit method has the disadvantage that it is only stable and convergent when the restriction $\Delta x>\sqrt{2 \Delta t}$ is imposed. This restriction implies that we can need many time steps to obtain the solution. This problem is avoided by the other two methods which are unconditionally stable and convergent although they require a more sophisticated calculation. ${ }^{7}$

Option valuation under finite difference methods is done by backward induction in time, as with lattices. Finite difference methods deal with European and

\footnotetext{
${ }^{6}$ The first two methods were introduced for option pricing in Schwartz (1977) and Brennan and Schwartz $(1977,1978)$ while the Crank-Nicolson method was first used in this framework by Courtadon (1982).

${ }^{7}$ In both cases, we have to solve a tridiagonal system of equations although it can be done in a very efficient way by using the Thomas algorithm. See Morton and Mayers (1998) for details.
} 
American derivatives, but it is difficult to extend them to path-dependent claims or options with multiple stochastic processes.

For two or three dimensions, LOD (Locally One Dimensional) and ADI (Alternating Direction Implicit) methods are developed. ${ }^{8}$ For higher dimensions, Monte Carlo simulation is required.

\subsubsection{Quadrature (Numerical Integration)}

American options can also be priced without approximating the stochastic process for the asset or the partial differential equation for the option price. This is the case of quadrature techniques, which are based on approximating a certain integral. Using an arbitrage argument, Karatzas (1988) shows that, for an European call option, the price $C_{t}$ at time $t \in[0, T]$ is given by

$$
C_{t}=E_{t}^{\tilde{P}}\left(e^{-r(T-t)} C_{T}\right)
$$

where $E_{t}$ is the expectation operator at time $t, \tilde{P}$ is the risk-neutral probability measure and $C_{T}$ is the value of this option at maturity (see eq. (4)). This call price formula can be rewritten as

$$
C_{t}=\int_{-\infty}^{\infty} e^{-r(T-t)} g(S) \max \left\{S_{T}-K, 0\right\} d S
$$

where $g(S)$ is the risk-neutral probability density function of the underlying asset. In general, this integral can only be solved numerically. This integral can be approximated by the sum of the integrand values at certain points, multiplied by some weighting coefficients. Examples of this technique are the trapezoidal and Simpson's rules.

Numerical integration is commonly used to price European derivatives, although Parkinson (1977) has used it for pricing American put options.

Some authors have worked with the so called "integral representation method". For instance, Kim (1990), Jacka (1991) and Carr et al. (1992) decompose the

\footnotetext{
${ }^{8}$ See Morton and Mayers (1998) for details.
} 
price of an American put into the price of an European put option plus the early exercise premium. This premium is expressed as an integral where the early exercise boundary is involved. Once this integral is computed, the American put price is easily obtained.

Different approximations of this integral have been proposed. Huang et al. (1996) approximate the integrand with step functions to decrease the number of early exercise points. After obtaining a sequence of approximated option prices, a four-point Richardson extrapolation is used to yield the American put price. $\mathrm{Ju}$ (1998) recognizes that the above integral does not depend critically on the early exercise boundary and uses a multipiece exponential function as an approximation of this boundary. This approximation jointly with the application of Richardson extrapolation lead to a closed-form expression for this integral. The approximation is shown to be exact in the extreme cases where the time to maturity goes to zero or to infinity. Numerical results show that this approximation together with the LUBA method in Broadie and Detemple (1996) and the randomization technique by Carr (1998) are the most accurate methods for pricing American options.

Recently, Bunch and Johnson (2000) have derived exact expressions for the critical stock price function and the American put price in the perpetual and finite cases. The key element of their derivation is that the critical stock price can be interpreted as the highest value of the stock price at which the put price does not depend on time to maturity. Finally, Ait-Sahlia and Lai (2000) propose two different solutions based on a piecewise linear approximation of the early exercise boundary.

\subsubsection{Monte Carlo Simulation}

This technique was introduced in finance by Boyle (1977). For a recent survey see Boyle et al. (1997). 
As shown in equation (6), the value of an option is the risk-neutral expectation of its discounted pay-off. This expectation is estimated by computing the average of a large number of pay-offs. These are the main steps to be followed:

1. Simulate the risk-neutral process for the price of the underlying asset (see eq. (1)) until maturity of the option and calculate the option pay-off. This step is repeated $M$ times.

2. Calculate the mean of these pay-offs.

3. Discount this average at the risk-free rate to obtain an estimate of the option value.

The crucial point is to simulate appropriately the path followed by the underlying asset. It is recommended to use the natural logarithm of the asset price. In this case, equation (2) is approximated by

$$
x(t+\Delta t)=x(t)+\nu \Delta t+\sigma \sqrt{\Delta t} \varepsilon, \quad \Delta t=T / N
$$

where $\varepsilon$ is extracted from a standard normal distribution. This equation is used to obtain the value of $x(t)$ along the path between zero and $T$.

Monte Carlo simulation is suitable for path-dependent options and can be extended to price option that depend on multiple stochastic processes, random volatility, jumps,.... Its major disadvantage is that it is a computationally intensive method, since it usually requires many simulations. With the aim of solving this problem, variance reduction techniques, such as antithetic variables and control variates, have been developed.

Tilley (1993) is the first who prices American options using this technique. He proposes an algorithm in which, at each date, simulated paths are ordered by asset price and bundled into groups. Then, for each group, an optimal exercise decision is taken. As Broadie and Glasserman (1997a) indicate, there are no convergence results for this algorithm, all the simulated paths must be stored at one time and there is not a direct extension to deal with multiple state variables. 
Barraquand and Martineau (1995) propose to reduce the dimensionality of the valuation problem, grouping the simulated values into a set of "bins". The transition probabilities between bins is determined by simulation and the option valuation is performed using each bin as a decision unit.

Broadie and Glasserman (1997a) present an algorithm that allows them to obtain point estimates and error bounds for American option prices. After showing that, under certain assumptions, there are not unbiased estimates of these prices, two (biased) estimates that converge asymptotically to the true price are generated. Combination of both estimates leads to a confidence interval for the American option price.

Broadie et al. (1997) and Raymar and Zwecher (1997) price American options on the maximum of several assets improving the techniques presented by Broadie and Glasserman (1997a) and Barraquand and Martineau (1995), respectively.

Ibañez and Zapatero (1998) suggest a general Monte Carlo simulation method for computing the optimal exercise frontier as the fixed point of an algorithm. To obtain this frontier, the values of all parameters but one are fixed and their algorithm is used to converge to the value of the remaining parameter in the optimal exercise frontier. Assuming that American derivatives can be exercised at a finite number of times, they price put options and call options on the maximum of two securities.

Finally, non-parametric methods can also be used to price American options. This is the case of neural networks that tries to recover an unknown pricing function given historical data. Once the network has "learned" from the data, it is applied to out of sample data to determine the unknown price. These methods allow us to price European and American derivatives with multiple stochastic processes. See, for example, Hutchinson et al. (1994). 


\section{The Least-Squares Monte Carlo Approach}

As mentioned before, the main problem for pricing American options is that there exist several possible exercise dates. Hence, the holder of the option must decide, at each exercise time, whether to exercise the option or to wait. This decision depends on the comparison between (a) the amount of money to be obtained if the option is exercised (the immediate exercise value) and (b) the amount of money he will obtain if the option is exercised at a future date (the continuation value).

Therefore, the optimal exercise decision relies on the estimation of the continuation value. Longstaff and Schwartz (2001) estimate this value by a least-squares regression jointly with the cross-sectional information provided by Monte Carlo simulation. In this regressions they use a set of basis functions whose arguments are based on the underlying asset prices. The fitted values of these regressions are taken as the expected continuation values. Comparing these estimated values with the immediate exercise ones, they identify the optimal stopping rule. This procedure is repeated recursively going back in time. Discounting the obtained cash-flows to time zero, the price of the American option is found.

More formally, they assume a finite time horizon, $[0, T]$, in which they define a probability space, ${ }^{9}(\Omega, \mathbb{F}, P)$, and an equivalent martingale measure, $Q$. Let $C(\omega, s ; t, T), \omega \in \Omega, s \in(t, T]$ denote the path of option cash-flows, conditional on (a) the option being exercised after $t$ and (b) the optionholder following the optimal stopping strategy at every time after $t$.

The American option is approximated by its Bermuda counterpart, assuming that there is a finite number of exercise dates $0<t_{1}<t_{2}<\ldots<t_{N}=T$. The continuation value is equal, under no-arbitrage conditions, to the risk-neutral

\footnotetext{
${ }^{9}$ This is a triple consisting of $\Omega$, the set of all possible sample paths $(\omega), \mathbb{F}$, the sigma-algebra of events at time $T$ and $P$, a probability measure defined on the elements of $\mathbb{F}$.
} 
expectation of the future discounted cash flows $C\left(\omega, s ; t_{i}, T\right)$ :

$$
F\left(\omega ; t_{i}\right)=E_{Q}\left[\sum_{j=i+1}^{N} \exp \left(-\int_{t_{i}}^{t_{j}} r(\omega, s) d s\right) C\left(\omega, t_{j} ; t_{i}, T\right) \mid \mathbb{F}_{t_{i}}\right]
$$

where $r(\omega, s)$ is the risk-free interest rate and $\mathbb{F}_{t_{i}}$ is the information set at time $t_{i}$.

The idea underlying the LSM algorithm is that this conditional expectation can be approximated by a least-squares regression for each exercise date. At time $t_{N-1}$, it is assumed that $F\left(\omega ; t_{N-1}\right)$ can be expressed as a linear combination of orthonormal basis functions $\left(p_{j}(X)\right)$ such as Laguerre, Hermite, Legendre or Jacobi polynomials. That is

$$
F\left(\omega ; t_{N-1}\right)=\sum_{j=0}^{\infty} a_{j} p_{j}(X), a_{j} \in \mathbb{R}
$$

that is approximated by

$$
F_{M}\left(\omega ; t_{N-1}\right)=\sum_{j=0}^{M} a_{j} p_{j}(X), a_{j} \in \mathbb{R} .
$$

This procedure is repeated going back in time until the first exercise date.

Longstaff and Schwartz (2001) apply their algorithm to price a number of American derivatives (American put option, American-Bermuda-Asian option, etc).

\subsection{A Numerical Example}

To provide numerical intuition, Longstaff and Schwartz (2001) present a numerical example. Here we include another numerical example that shows that, if we use the LSM approach with a reduced number of simulated paths, an American option can have a lower price than its European counterpart.

We price an American put option on a non-dividend stock. The strike price is 1.1 and there are three possible exercise dates. The continuously compounded risk-free interest rate is equal to 0.05 . 
We simulate eight paths of the underlying stock price as shown in the following table $^{10}$

\begin{tabular}{ccllll} 
Path & $\mathrm{t}=0$ & $\mathrm{t}=1$ & $\mathrm{t}=2$ & $\mathrm{t}=3$ & Pay-off at $\mathrm{t}=3$ \\
\hline 1 & 1 & $0.917938^{*}$ & 1.272171 & 1.417021 & 0 \\
2 & 1 & 1.133931 & 1.290983 & 1.669802 & 0 \\
3 & 1 & 1.162833 & $0.917742 *$ & 1.228432 & 0 \\
4 & 1 & $1.096706 *$ & $1.081163 *$ & 1.118280 & 0 \\
5 & 1 & $1.056690 *$ & $0.871784^{*}$ & $0.818722 *$ & 0.281278 \\
6 & 1 & 1.416442 & 1.672474 & 1.263264 & 0 \\
7 & 1 & $0.937138 *$ & $0.945920^{*}$ & $0.861259 *$ & 0.238741 \\
8 & 1 & $0.872576 *$ & $0.658605^{*}$ & $0.475270 *$ & 0.624730 \\
\hline
\end{tabular}

The last column of this table shows the final pay-offs of an European option. Discounting these pay-offs at time zero and averaging them, we obtain that the price of this European option is equal to 0.123162 .

For an American option, the LSM approach maximizes its value at each exercise date along in-the-money (ITM) paths. For each date, $X$ denotes the underlying price and $Y$ represents the (discounted) cash-flows received at future dates if the option is not exercised.

At time two, there are five ITM paths (all but the first, the second and the sixth ones) and the values of $X$ and $Y$ are as follows

\footnotetext{
${ }^{10}$ The symbol ${ }^{*}$, denotes the in-the-money paths. Focusing on this type of paths improves the efficiency of the LSM method.
} 


\begin{tabular}{ccc} 
Path & $Y$ & $X$ \\
\hline 1 & - & - \\
2 & - & - \\
3 & $e^{-0.05} \times 0$ & 0.917742 \\
4 & $e^{-0.05} \times 0$ & 1.081163 \\
5 & $e^{-0.05} \times 0.281278$ & 0.871784 \\
6 & - & - \\
7 & $e^{-0.05} \times 0.238741$ & 0.945920 \\
8 & $e^{-0.05} \times 0.624730$ & 0.658605 \\
\hline
\end{tabular}

To decide whether to exercise or not, we must estimate the continuation value and compare it with the immediate exercise value, $1.1-X$. The continuation value is estimated by regressing $Y$ on a constant, $X$ and $X^{2}$, which gives

$$
E[Y \mid X]=2.848474-4.6539 X+1.871826 X^{2}
$$

and, then, the exercise decision are as follows

\begin{tabular}{cccc} 
Path & $1.1-X$ & $E[Y \mid X]$ & Decision \\
\hline 1 & - & - & - \\
2 & - & - & - \\
3 & 0.182258 & 0.1539056 & Exercise \\
4 & 0.018837 & 0.0048106 & Exercise \\
5 & 0.228216 & 0.2138467 & Exercise \\
6 & - & - & - \\
7 & 0.154080 & 0.1210645 & Exercise \\
8 & 0.441395 & 0.5952915 & Wait \\
\hline
\end{tabular}

In this table, we see that we exercise the option in all the ITM paths except the eighth one, in which $1.1-X<E[Y \mid X]$. Therefore, assuming that the option is not exercised before time two, the cash-flows to the optionholder are the following 


\begin{tabular}{llll} 
Path & $\mathrm{t}=1$ & $\mathrm{t}=2$ & $\mathrm{t}=3$ \\
\hline 1 & - & 0 & 0 \\
2 & - & 0 & 0 \\
3 & - & 0.182258 & 0 \\
4 & - & 0.018837 & 0 \\
5 & - & 0.228216 & 0 \\
6 & - & 0 & 0 \\
7 & - & 0.154080 & 0 \\
8 & - & 0 & 0.62473 \\
\hline
\end{tabular}

We repeat this procedure at time one, when we also have five ITM paths. Now, to compute the variable $Y$, we use the cash-flows to be received at time two or three (but not in both dates) for each path. The values of $X$ and $Y$ are as shown next

\begin{tabular}{ccc} 
Path & $Y$ & $X$ \\
\hline 1 & $e^{-0.05} \times 0$ & 0.917938 \\
2 & - & - \\
3 & - & - \\
4 & $e^{-0.05} \times 0.018837$ & 1.096706 \\
5 & $e^{-0.05} \times 0.228216$ & 1.056690 \\
6 & & \\
7 & $e^{-0.05} \times 0.154080$ & 0.937138 \\
8 & $\left(e^{-0.05}\right)^{2} \times 0.624730$ & 0.872576 \\
\hline
\end{tabular}

Estimating again $Y$ on a constant and the first two powers of $X$, we obtain

$$
E[Y \mid X]=23.905695-47.1482 X+23.23217 X^{2}
$$

which leads us to the following exercise decision 


\begin{tabular}{cccl} 
Path & $1.1-X$ & $E[Y \mid X]$ & Decision \\
\hline 1 & 0.182062 & 0.202191 & Wait \\
2 & - & - & - \\
3 & - & - & - \\
4 & 0.003294 & 0.1407488 & Wait \\
5 & 0.043310 & 0.0255102 & Exercise \\
6 & - & - & - \\
7 & 0.162862 & 0.1244155 & Exercise \\
8 & 0.227424 & 0.4539830 & Wait \\
\hline
\end{tabular}

Consequently, the cash-flows paid by this American option at the three exercise dates are the following

\begin{tabular}{clll} 
Path & $\mathrm{t}=1$ & $\mathrm{t}=2$ & $\mathrm{t}=3$ \\
\hline 1 & 0 & 0 & 0 \\
2 & 0 & 0 & 0 \\
3 & 0 & 0.182258 & 0 \\
4 & 0 & 0.018837 & 0 \\
5 & 0.043310 & 0 & 0 \\
6 & 0 & 0 & 0 \\
7 & 0.162862 & 0 & 0 \\
8 & 0 & 0 & 0.62473 \\
\hline
\end{tabular}

Thus, at time one, we exercise the option in the fifth and seventh paths. At time two, we exercise the option in the third and the fourth paths and, at time three, a non-zero cash-flow is received in the eighth path.

Obviously, all the cash-flows in the second and the sixth paths are null because they are out-of-the money paths. For the first path, the cash-flows are also zero even though, at time one, the option is ITM. This can be explained because the optimal decision at this time was to wait. 
Finally, discounting these cash-flows to the initial date and averaging them over all paths, we obtain that the price for the American option is 0.114473 , a $7 \%$ smaller than the corresponding European option. Of course, this is a consequence of the reduced number of simulated paths. Increasing the number of simulated paths leads to American option prices that are larger than European ones.

\section{Numerical Results on the Robustness of LSM}

An interesting question is to analyze what happens when we change the number of terms of the basis functions or we use alternative functions. In this paper, we use ten different polynomials with up to ten terms as basis functions. ${ }^{11}$

\begin{tabular}{lc} 
Name & $f_{n}(x)$ \\
\hline Powers & $W_{n}(x)$ \\
Legendre & $P_{n}(x)$ \\
Laguerre & $L_{n}(x)$ \\
Hermite A & $H_{n}(x)$ \\
Hermite B & $H_{e_{n}}(x)$ \\
Chebyshev 1st kind A & $T_{n}(x)$ \\
Chebyshev 1st kind B & $C_{n}(x)$ \\
Chebyshev 1st kind C & $T_{n}^{*}(x)$ \\
Chebyshev 2nd kind A & $U_{n}(x)$ \\
Chebyshev 2nd kind B & $S_{n}(x)$ \\
\hline
\end{tabular}

These polynomials can be expressed in three alternative ways:

1. Rodrigues' formula:

$$
f_{n}(x)=\frac{1}{a_{n} g(x)} \frac{\partial^{n}}{\partial x^{n}}\left[\rho(x)(g(x))^{n}\right]
$$

\footnotetext{
${ }^{11}$ See Demidowitsch et al. (1980) for details on $T_{n}^{*}(x)$ and Abramowitz and Stegun (1972) for the remaining ones.
} 
2. Explicit expression:

$$
f_{n}(x)=d_{n} \sum_{m=0}^{N} c_{m} g_{m}(x)
$$

3. Recurrence law:

$$
a_{n+1} f_{n+1}(x)=\left(a_{n}+b_{n} x\right) f_{n}(x)-a_{n-1} f_{n-1}(x)
$$

The coefficients and functions included in these expressions are shown in Tables 1,2 , and 3 , respectively.

$$
\text { [ Insert Tables 1, 2, and } 3 \text { about here ] }
$$

From a theoretical point of view, it would be desirable to use an orthonormal basis of functions on which to project continuation values. This means that

$$
\int_{a}^{b} f_{n}(x) f_{m}(x) d x=\left\{\begin{array}{cc}
0 & n \neq m \\
1 & n=m
\end{array}\right.
$$

The values for the limits of this integral vary with the polynomials. See Abramowitz and Stegun (1972) for details. In most of the cases, the range of underlying prices $(X)$ is different from the interval $[a, b]$ so that the basis functions will no be orthonormal. Consequently, we should increase the number of terms used in the regression.

\subsection{Valuation of the Standard Put Option}

The first derivative we price is an American put option on a non-dividend stock with the following characteristics: $\sigma=0.2, r=0.06, T=1, S_{0}=K=40$. We approximate this option, assuming that there are 70 exercise dates.

The value of the American option using the binomial method of Cox et al. (1979) (with 1.000 iterations) is 2.31928. The value of the corresponding European option, using simulation and the Black and Scholes (1973) formula, are 2.06193 and 2.06640, respectively. 
To avoid numerical problems, we standardize the option dividing by the strike price and we use double-precision variables. We also employ the routine SVDFIT, a Numerical Recipes routine that performs linear least-squares fits using the singular value decomposition technique.

Results for the LSM algorithm with different basis functions and number of terms are shown in Table 4. We use 100.000 simulations, half of them with antithetic variables. Notice that this implies that we have to store $(100.000 \times 70)$ matrices.

\section{[ Insert Table 4 about here ]}

For the ten polynomials, we obtain similar prices. We see that, typically, the option value increases with the number of terms. However, using more than four terms does not change significantly the prices. This result is common for all the polynomials. Notice that the computed option prices are lower than the value obtained with the binomial method. This is not surprising since we are considering only 70 exercise dates.

\subsection{Option on the maximum of five assets}

We now turn our attention to a more sophisticated derivative: an American call option on the maximum of five uncorrelated assets. The volatility of the asset returns is taken as 0.2 , the interest rate is 0.05 , the dividend yield is 0.1 , the maturity of the option is three years, and there are three exercise times per year. The strike price is 100 and the initial assets prices are also 100 for the five assets.

This option has been priced by Broadie and Glasserman (1997b), using the stochastic mesh method. They find that the $90 \%$ confidence interval for the price of this option is [26.101, 26.211].

Longstaff and Schwartz (2001) also value this option using the LSM approach with 19 basis functions. These functions are a constant, five Hermite polynomials in the maximum of the five assets, the second to the fifth maximums and 
their square values, the four products of consecutive pairs of maximums, and the product of the five assets. Using 50.000 paths, they obtain that the value of the option is 26.182, which is within the interval given by Broadie and Glasserman (1997b).

As before, we use different basis functions to price this option. In Table 5, we show option prices obtained when the Hermite polynomial is replaced by other polynomials, with up to ten terms. This means that we use between 14 and 24 basis functions. We simulate $50.000+50.000$ antithetic paths.

\section{[ Insert Table 5 about here ]}

We see that using less than two terms, we obtain values which are outside the interval given by Broadie and Glasserman (1997b). We also obtain values outside this interval using more than seven terms for the polynomials $P_{n}(x), H_{n}(x), T_{n}(x)$ and $U_{n}(x)$. For the remaining cases, the option values are inside the interval, and increase up to four to five terms. For all the polynomials, using more terms, option prices decrease (in some cases, these prices are lower than 24.0). This result is against Proposition 1 in Longstaff and Schwartz (2001), that predicts that option prices increase with the number of terms of basis functions. A final remark is that using five Hermite polynomials, the option price is 26.187 , which is very close to the price given by Longstaff and Schwartz (2001).

As a reference, the value of the corresponding European option is 23.098, so that the early exercise premium is higher than 3 .

Now, we set the basis functions equal to $H_{e_{n}}(x)$, and we change the remaining ones. The results are shown in Table 6 .

\section{[ Insert Table 6 about here ]}

The second column presents the prices obtained without including the square values of the second to the fifth maximums. We see that dropping out those values has little impact on the option price. As before, the option value increases 
and then decreases with the number of terms. In the third column, we also drop out the products of consecutive maximums. We observe that, in this case, option prices are outside the interval, except when we use five terms. In the following column, we work with the polynomials $H_{e_{n}}(x)$, the second to the fifth maximums, and their square values. Now, all the option prices are outside the interval. Finally, the fifth column shows the prices obtained with the same basis functions as in Table 5 plus the third powers of the second to the fifth maximums. Compared to the sixth column of Table 5, we find that option prices are similar in both cases.

\section{Conclusions}

Monte Carlo simulation is widely used for pricing European options. However, application of this technique for valuing American derivatives is not straightforward.

Recently, Longstaff and Schwartz (2001) have developed the Least-Squares Monte Carlo (LSM) technique, that uses simple regressions to price American options. At each exercise date, they estimate the continuation value of the option regressing the expected cash-flows on basis functions of the underlying asset price.

In this paper, we analyze the robustness of the LSM approach to the choice of basis functions and to the number of terms used. We apply the algorithm to price an American put option and an American call option on the maximum of five assets. We consider ten different basis functions and up to ten terms. Numerical results show that the technique is very robust for the simplest (American put) case. However, for high-dimensional problems, the robustness does not seem to be guaranteed and the choice of the basis functions to be used is not clear. 


\section{References}

[1] Abramowitz, W. and I. Stegun (1972). Handbook of Mathematical Functions with Formulas, Graphs, and Mathematical Tables, Dover Publications, Inc., New York.

[2] Ait-Sahlia, F. (1996). Optimal Stopping and Weak Convergence Methods for Some Problems in Financial Economics. Ph.D. Dissertation, Dept. of Operations Research, Stanford University.

[3] Ait-Sahlia, F. and P. Carr (1997). American Options: A Comparison of Numerical Methods. In Numerical Methods in Finance, edited by L.C.G. Rogers and D. Talay, Cambridge University Press.

[4] Ait-Sahlia, F. and T.L. Lai (1996). Approximations for American Options, working paper, Cornell University.

[5] (2000). A Canonical Optimal Stopping Problem for American Options and its Numerical Solution. Journal of Computational Finance, 3, 2, 33-52.

[6] Barone-Adesi, G. and R.E. Whaley (1987). Efficient Analytic Approximation of American Option Values. Journal of Finance, 42, 2, 301-320.

[7] Barraquand, J. and D. Martineau (1995). Numerical Valuation of High Dimensional Multivariate American Securities. Journal of Financial and Quantitative Analysis, 30, 383-405.

[8] Black, F. and M. Scholes (1973). The Pricing of Options and Corporate Liabilities. Journal of Political Economy, 81, 3, 637-654.

[9] Boyle, P. (1977). Options: A Monte Carlo Approach. Journal of Financial Economics, 4, 323-338. 
[10] Boyle, P. (1988). A Lattice Framework for Option Pricing with Two State Variables. Journal of Financial and Quantitative Analysis. 22, $1-12$.

[11] Boyle, P., M. Broadie and P. Glasserman (1997). Monte Carlo Methods for Security Pricing. Journal of Economic Dynamics and Control, 21, 1267-1321.

[12] Breen, R. (1991). The Accelerated Binomial Option Pricing Model. Journal of Financial and Quantitative Analysis, 26, 153-164.

[13] Brennan, M.J. and E.S. Schwartz (1977). The Valuation of American Put Options. Journal of Finance, 32, 449-462.

[14] (1978). Finite Difference Methods and Jump Processes Arising in the Pricing of Contingent Claims: A Synthesis. Journal of Financial and Quantitative Analysis, 13, 461-474.

[15] Broadie, M. and J. Detemple (1996). American Option Valuation: New Bounds, Approximations and a Comparison of Existing Methods. Review of Financial Studies, 9, 1211-1250.

[16] Broadie, M. and P. Glasserman (1997a). Pricing American-Style Securities using Simulations. Journal of Economic Dynamics and Control, 21, 1323-1352.

[17] (1997b). A Stochastic Mesh Method for Pricing HighDimensional American Options, working paper, Columbia University.

[18] Broadie, M., P. Glasserman and G. Jain (1997). Enhanced Monte Carlo estimation for American Option Prices. Journal of Derivatives, $5,1,25-44$. 
[19] Bunch, D. and H.E. Johnson (1992). A Simple and Numerically Efficient Valuation Method for American Puts Using a Modified Geske-Johnson Approach. Journal of Finance, 47, 2, 809-816.

[20] (2000). The American Put Option and Its Critical Stock Price. Journal of Finance, 55, 5, 2333-2356.

[21] Carr, P. (1998). Randomization and the American Put. Review of Financial Studies, 11, 597-626.

[22] Carr, P. and D. Faguet (1996). Fast Accurate Valuation of American Options, working paper, Cornell University.

[23] Carr, P., R. Jarrow and R. Mynemi (1992). Alternative Characterization of American Puts. Mathematical Finance, 2, 87-106.

[24] Clewlow, L. and C. Strickland (1998), Implementing Derivatives Models, John Wiley \& Sons Ltd., England.

[25] Courtadon (1982). A More Accurate Finite Difference Approximation for the Valuation of Options. Journal of Financial and Quantitative Analysis, 17, 5, 697-703.

[26] Cox, J.C., S.A. Ross and M. Rubinstein (1979). Option Pricing: A Simplified Approach. Journal of Financial Economics, 7, 3, 229-263.

[27] Demidowitsch, B.P., I.A. Maron and E.S. Schuwalowa (1980). Métodos numéricos de análisis. Ed. Paraninfo, S.A. Madrid.

[28] Geske, R. (1979). A Note on an Analytical Valuation Formula for Unprotected American Options on Stocks with Known Dividends. Journal of Financial Economics, 7, 375-380.

[29] Geske, R. and H.E. Johnson (1984). The American Put Option Valued Analytically. Journal of Finance, 39, 5, 1511-1524. 
[30] Ho, T.S.Y., R.C. Stapleton and M.G. Subrahmanyam (1994). A Simple Technique for the Valuation and Hedging of American Options. Journal of Derivatives, 2, 52-66.

[31] - (1997). The Valuation of American Options with Stochastic Interest Rates: A Generalization of the Geske-Johnson Technique. Journal of Finance, 52, 2, 827-840.

[32] Huang, J.Z., M.G. Subrahmanyam and G.G. Yu (1996). Pricing and Hedging American Options: A Recursive Integration Method. Review of Financial Studies, 9, 1, 277-300.

[33] Hutchinson, J., A. Lo and T. Poggio (1994). A Nonparametric Approach to Pricing and Hedging Derivative Securities. Journal of Finance, 49, 3, 851-886.

[34] Ibañez, A. and F. Zapatero (1998). Monte Carlo Valuation of American Options through Computation of the Optimal Exercise Frontier, working paper, The University of Southern California.

[35] Jacka, S.D. (1991). Optimal Stopping and the American Put. Mathematical Finance, 1, 1-14.

[36] Jarrow, R. and A. Rudd (1983). Option Pricing. Dow Jones-Irwin, Homewood, Illinois.

[37] Johnson, H.E. (1983). An Analytic Approximation for the American Put Price. Journal of Financial and Quantitative Analysis, 18, 1, 141-148.

[38] Ju, N. (1998). Pricing American Option by Approximating its Early Exercise Boundary as a Multipiece Exponential Function. Review of Financial Studies, 11, 3, 627-646. 
[39] Karatzas, I. (1988). On the Pricing of American Options. Applied Mathematics and Optimization, 17, 37-60.

[40] Kim, I.J. (1990). The Analytical Valuation of American Options. Review of Financial Studies, 3, 547-572.

[41] Longstaff, F.A. and E.S. Schwartz (2001). Valuing American Options by Simulations: A Simple Least-Squares Approach. Review of Financial Studies, 14, 1, 113-147.

[42] McKean, H.P. (1965). Appendix: A Free Boundary Problem for the Heat Equation Arising from a Problem in Mathematical Economics. Industrial Management Review, 6, 32-39.

[43] Merton, R.C. (1973). Theory of Rational Option Pricing. Bell Journal of Economics and Management Science, 4, 1, 141-183.

[44] Morton, K.W. and D.F. Mayers (1998). Numerical Solution of Partial Differential Equations. Cambridge University Press.

[45] Parkinson, M. (1977). Option Pricing: The American Put. Journal of Business, 50, 21-36.

[46] Raymar, S. and M. Zwecher (1997). Monte Carlo Estimation of American Call Options on the Maximum of Several Stocks. Journal of Derivatives, 5, 7-24.

[47] Rektorys, K. (1982). The Method of Discretization in Time and Partial Differential Equations, D. Reidel Publishing, Boston, Mass.

[48] Rendleman, R. and B. Bartter (1979). Two-State Option Pricing. Journal of Finance, 34, 1093-1110. 
[49] Roll, R. (1977). An Analytic Valuation Formula for Unprotected American Call Options on Stocks with Known Dividends. Journal of Financial Economics, 5, 251-258.

[50] Schwartz, E.S. (1977). The Valuation of Warrants: Implementing a New Approach. Journal of Financial Economics, 4, 79-93.

[51] Tilley, J. (1993). Valuing American Options in a Path Simulation Model. Transactions of the Society of Actuaries, 45, 83-104.

[52] Whaley, R.E. (1981). On the Valuation of American Call Options on Stocks whit Known Dividends. Journal of Financial Economics, 9, 2, 207-211. 
Table 1: Expressions of the basis functions using Rodrigues formula.

\begin{tabular}{cccc}
$f_{n}(x)$ & $a_{n}$ & $\rho(x)$ & $g(x)$ \\
\hline$W_{n}(x)$ & $\frac{(2 n) !}{n !}$ & $x^{2 n}$ & 1 \\
$P_{n}(x)$ & $(-1)^{n} 2^{n} n !$ & 1 & $1-x^{2}$ \\
$L_{n}(x)$ & $n !$ & $e^{-x}$ & $x$ \\
$H_{n}(x)$ & $(-1)^{n}$ & $e^{-x^{2}}$ & 1 \\
$H_{e_{n}}(x)$ & $(-1)^{n}$ & $e^{-x^{2} / 2}$ & 1 \\
$T_{n}(x)$ & $(-1)^{n} 2^{n} \frac{\Gamma\left(n+\frac{1}{2}\right)}{\sqrt{\pi}}$ & $\left(1-x^{2}\right)^{-1 / 2}$ & $1-x^{2}$ \\
$C_{n}(x)$ & $(-1)^{n} 2^{n} \frac{\Gamma\left(n+\frac{1}{2}\right)}{\sqrt{\pi}}$ & $\left(1-\frac{x^{2}}{4}\right)^{-1 / 2}$ & $1-\frac{x^{2}}{4}$ \\
$T_{n}^{*}(x)$ & $(-1)^{n} 2^{2 n-1} \frac{\Gamma\left(n+\frac{1}{2}\right)}{\sqrt{\pi}}$ & $\left(1-x^{2}\right)^{-1 / 2}$ & $1-x^{2}$ \\
$U_{n}(x)$ & $\frac{(-1)^{n} 2^{n+1} \Gamma\left(n+\frac{3}{2}\right)}{(n+1) \sqrt{\pi}}$ & $\left(1-x^{2}\right)^{1 / 2}$ & $1-x^{2}$ \\
$S_{n}(x)$ & $\frac{(-1)^{n} 2^{n+1} \Gamma\left(n+\frac{3}{2}\right)}{(n+1) \sqrt{\pi}}$ & $\left(1-\frac{x^{2}}{4}\right)^{1 / 2}$ & $1-\frac{x^{2}}{4}$ \\
\hline
\end{tabular}

The basis functions are especial cases of Rodrigues' formula which is given by

$$
f_{n}(x)=\frac{1}{a_{n} g(x)} \frac{\partial^{n}}{\partial x^{n}}\left[\rho(x)(g(x))^{n}\right]
$$

where $n \geq 0$ denotes the degree of the polynomial. 
Table 2: Explicit expressions of the basis functions.

\begin{tabular}{ccccc}
$f_{n}(x)$ & $N$ & $d_{n}$ & $c_{m}$ & $g_{m}(x)$ \\
\hline$W_{n}(x)$ & 0 & 1 & 1 & $x^{n}$ \\
$P_{n}(x)$ & {$[n / 2]$} & $2^{-n}$ & $(-1)^{m}\left(\begin{array}{c}n \\
m\end{array}\right)\left(\begin{array}{c}2 n-2 m \\
n\end{array}\right)$ & $x^{n-2 m}$ \\
& & & $\frac{(-1)^{m}}{m !}\left(\begin{array}{c}n \\
n-m\end{array}\right)$ & $x^{m}$ \\
$L_{n}(x)$ & $n$ & 1 & $(-1)^{m} \frac{1}{m !(n-2 m) !}$ & $(2 x)^{n-2 m}$ \\
$H_{n}(x)$ & {$[n / 2]$} & $n !$ & $(-1)^{m} \frac{1}{m !(n-2 m) !}$ & $x^{n-2 m}$ \\
$H_{e_{n}}(x)$ & {$[n / 2]$} & $n !$ & $(-1)^{m} \frac{(n-m-1) !}{m !(n-2 m) !}$ & $(2 x)^{n-2 m}$ \\
$T_{n}(x)$ & {$[n / 2]$} & $n / 2$ & $(-1)^{m} \frac{(n-m-1) !}{m !(n-2 m) !}$ & $x^{n-2 m}$ \\
$C_{n}(x)$ & {$[n / 2]$} & $n$ & $(-1)^{m} \frac{(n-m-1) !}{m !(n-2 m) !}$ & $(2 x)^{n-2 m}$ \\
$T_{n}^{*}(x)$ & {$[n / 2]$} & $2^{-n} n$ & $(-1)^{m} \frac{(n-m) !}{m !(n-2 m) !}$ & $(2 x)^{n-2 m}$ \\
$U_{n}(x)$ & {$[n / 2]$} & 1 & $(-1)^{m} \frac{(n-m) !}{m !(n-2 m) !}$ & $x^{n-2 m}$ \\
$S_{n}(x)$ & {$[n / 2]$} & 1 &
\end{tabular}

The basis functions are particular cases of the following expression

$$
f_{n}(x)=d_{n} \sum_{m=0}^{N} c_{m} g_{m}(x)
$$

where $n \geq 0$ denotes the degree of the polynomial. 
Table 3: Recurrence law for the basis functions.

\begin{tabular}{ccccccc}
$f_{n}(x)$ & $a_{n+1}$ & $a_{n}$ & $b_{n}$ & $a_{n-1}$ & $f_{0}(x)$ & $f_{1}(x)$ \\
\hline$W_{n}(x)$ & 1 & 0 & 1 & 0 & 1 & $x$ \\
$P_{n}(x)$ & $n+1$ & 0 & $2 n+1$ & $n$ & 1 & $x$ \\
$L_{n}(x)$ & $n+1$ & $2 n+1$ & -1 & $n$ & 1 & $1-x$ \\
$H_{n}(x)$ & 1 & 0 & 2 & $2 n$ & 1 & $2 x$ \\
$H_{e_{n}}(x)$ & 1 & 0 & 1 & $n$ & 1 & $x$ \\
$T_{n}(x)$ & 1 & 0 & 2 & 1 & 1 & $x$ \\
$C_{n}(x)$ & 1 & 0 & 1 & 1 & 2 & $x$ \\
$T_{n}^{*}(x)$ & 1 & 0 & 1 & $1 / 4$ & 1 & $x$ \\
$U_{n}(x)$ & 1 & 0 & 2 & 1 & 1 & $2 x$ \\
$S_{n}(x)$ & 1 & 0 & 1 & 1 & 1 & $2 x$ \\
\hline
\end{tabular}

The general expression for the recurrence law is given by

$$
a_{n+1} f_{n+1}(x)=\left(a_{n}+b_{n} x\right) f_{n}(x)-a_{n-1} f_{n-1}(x)
$$




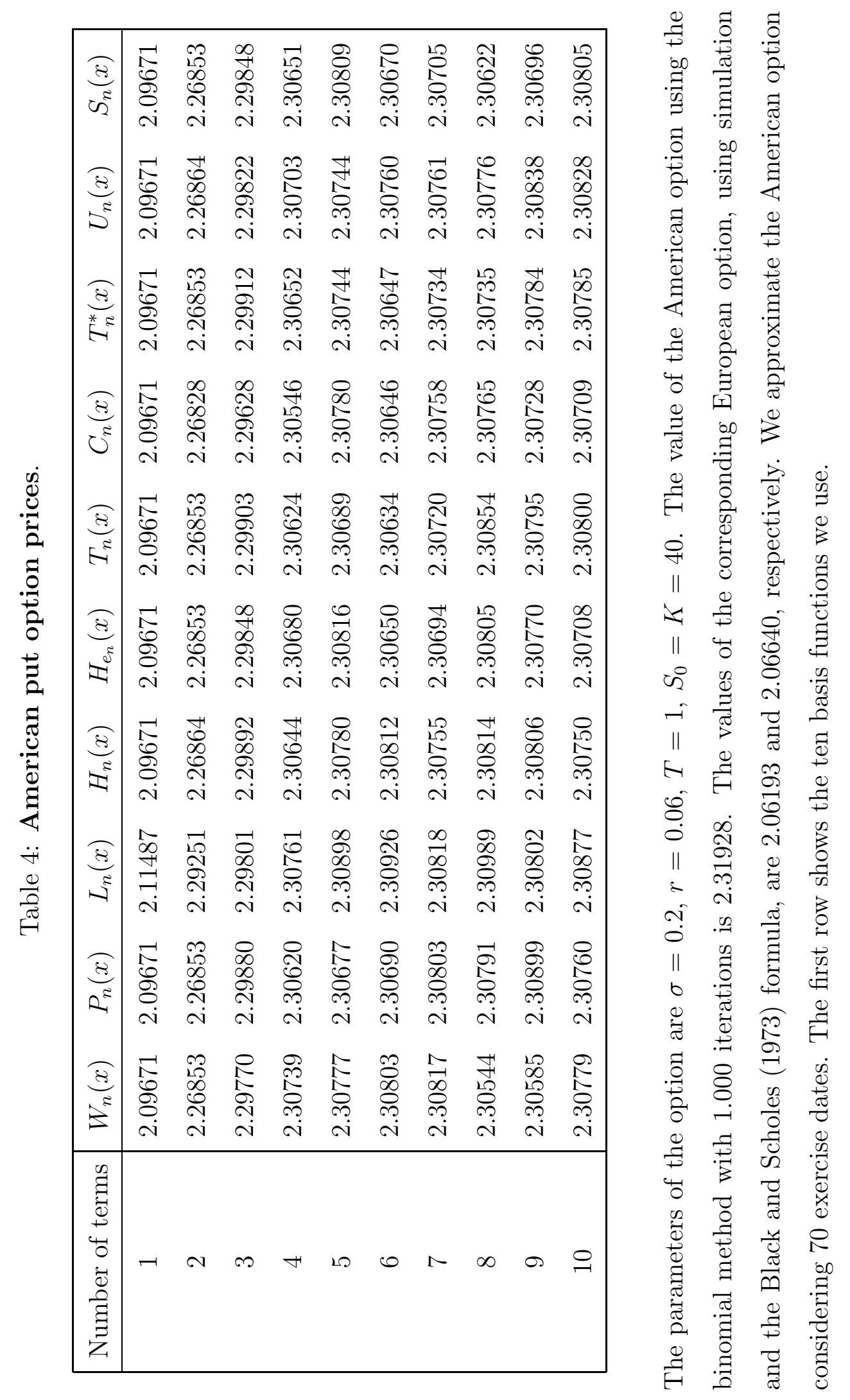




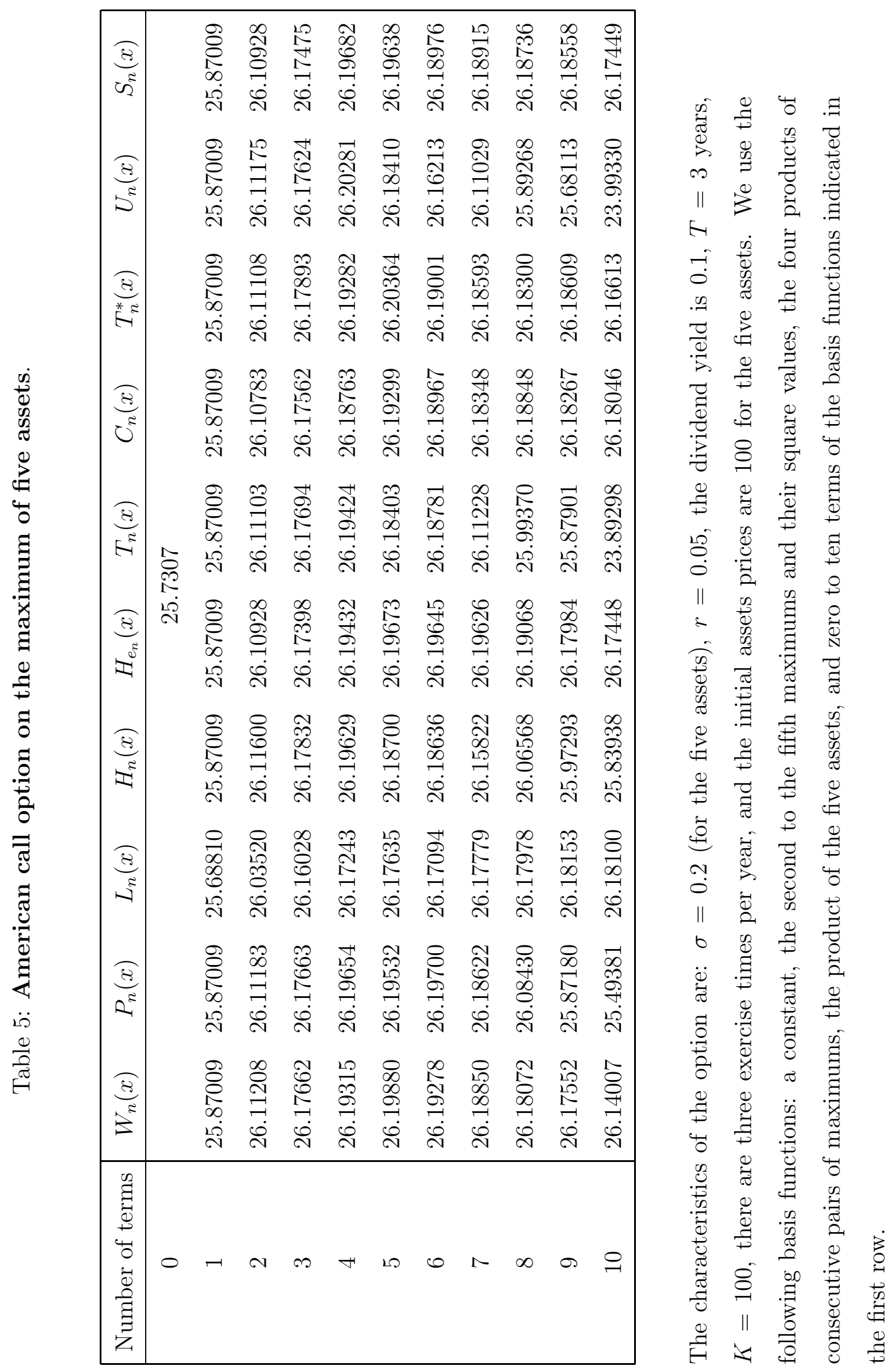


Table 6: Effect of basis functions on the American call option on the maximum of five assets.

\begin{tabular}{|c|cccc|}
\hline Number of terms & Case I & Case II & Case III & Case IV \\
\hline 0 & 25.75969 & 25.66929 & 25.36842 & 25.76960 \\
1 & 25.86988 & 25.76343 & 25.84218 & 25.88012 \\
2 & 26.07051 & 26.03418 & 26.05393 & 26.10515 \\
3 & 26.15420 & 26.06087 & 26.06920 & 26.18469 \\
4 & 26.16086 & 26.08385 & 26.09032 & 26.19798 \\
5 & 26.16341 & 26.10152 & 26.08691 & 26.20064 \\
6 & 26.14974 & 26.08556 & 26.07915 & 26.18932 \\
7 & 26.15517 & 26.09288 & 26.07881 & 26.19892 \\
8 & 26.14555 & 26.09031 & 26.07253 & 26.18422 \\
9 & 26.14921 & 26.08191 & 26.07572 & 26.17811 \\
10 & 26.11754 & 26.08579 & 26.07383 & 26.18058 \\
\hline
\end{tabular}

The characteristics of the option are: $\sigma=0.2$ (for the five assets), $r=0.05$, the dividend yield is $0.1, T=3$ years, $K=100$, there are three exercise times per year, and the initial assets prices are 100 for the five assets. In Case I we use the following basis functions: a constant, the second to the fifth maximums, the four products of consecutive pairs of maximums, and the product of the five assets. Case II is the same as Case I but without the products of consecutive maximums. Case III considers the second to the fifth maximums and their square values. Finally, Case IV uses a constant, the second to the fifth maximums, their square values, their third powers, the products of consecutive pairs of maximums, and the product of the five assets. In all the cases, we also use the polynomials $H_{e_{n}}(x)$ with up to ten terms. 